7th International Workshop on Astronomy and

Relativistic Astrophysics (IWARA 2016)

International Journal of Modern Physics: Conference Series

Vol. 45 (2017) 1760028 (4 pages)

(C) The Author(s)

DOI: $10.1142 / \mathrm{S} 201019451760028 \mathrm{X}$

\title{
Combustion of Hadrons to Strange Quark Matter Inside a Neutron Star
}

\author{
Jhon Andersson Rosero and Ernesto Kemp \\ Instituto de Física Gleb Wataghi \\ Universidade Estadual de Campinas (UNICAMP) \\ Rua Sérgio Buarque de Holanda, 777, 13083-859 Campinas, Brazil \\ jarosero@ifi.unicamp.br,kemp@ifi.unicamp.br
}

Published 15 August 2017

\begin{abstract}
We have studied the phase transition from hadronic to quark matter inside neutron stars, we calculate the rate and emissivity for all the relevant weak interaction processes and solve the Boltzmann transport equation, considering the effect of strong interactions in the perturbative regime to the order of QCD coupling constant $\alpha_{c}$. We find that the neutrino and antineutrino emissivity is around of $10^{53} \mathrm{erg}$.
\end{abstract}

Keywords: Stars, Quark Matter, Combustion.

PACS numbers: 12.39.-x, 24.85.+p, 05.70.-a, 97.10.-q

\section{Introduction}

Neutron stars (NS) are remnants of massive stars formed in core collapse supernova explosion, and these stars are cold objects that have lived long enough to get rid of their initial neutrino content, in other words, with $\mu_{\nu_{e}}=0$ where $\mu_{\nu_{e}}$ is the electron neutrino chemical potential. The strongly interacting matter at high densities and temperatures undergoes a phase transition from confined hadronic matter to quark matter. ${ }^{1,2}$ This transition may occur in a variety of physically different situations such as those found in heavy ion collisions and astrophysics.

We consider this problem in the astrophysical context and according to theoretical studies, the formation of quark matter in NS with the nucleation of small deconfined drops inside the neutron star core when the density of hadronic matter goes beyond a critical density. The formation of such drops occurs in two steps: First, the hadronic matter is deconfine in a strong interaction timescale of $\sim 10^{-23}$ seconds to quark matter, with result a quark gas which is not in equilibrium. Then, weak interactions will chemically equilibrate the system in a timescale of $\sim 10^{-8}$

This is an Open Access article published by World Scientific Publishing Company. It is distributed under the terms of the Creative Commons Attribution 4.0 (CC-BY) License. Further distribution of this work is permitted, provided the original work is properly cited. 
sec. As a result of these decays, quark matter in chemical equilibrium is produced, temperature is significantly increased, and a great amount of antineutrinos and neutrinos are produced.

The energy released in such conversion can ignite hadronic matter in the neighborhood of the drop, therefore it may grow and star core convert to quark matter and it is possible that the whole star if quark matter is absolutely stable. During the process, a combustion front (flame) is separating the burnt quark matter that travels outwards along the star from the unburnt hadronic matter. ${ }^{3}$ In this work we are interested in how quark matter within the flame approaches to equilibrium after the deconfinement transition inside a NS. The more relevant processes in a $\mathrm{NS}$ are $d \rightarrow u+e^{-}+\bar{\nu}_{e}, s \rightarrow u+e^{-}+\bar{\nu}_{e}, u+e^{-} \rightarrow d+\nu_{e}, u+e^{-} \rightarrow s+\nu_{e}$ and $u+d \leftrightarrow u+s$.

In Sec. 2 we give the rates and the emissivities for all the relevant processes. In Sec. 3 we solve the Boltzmann transport equation and describe the time evolution of the system as it approximate chemical equilibrium.

\section{Weak Reaction Rates and Emissivities}

The reaction rates and emissivities have been calculated in the literature ${ }^{4-6}$ where quarks and electrons are considered degenerate inside NS. In this work, we obtain the reaction rates and emissivities assuming degenerate quarks and electrons, but without making any assumption about the degeneracy state of neutrinos or antineutrinos. Due to space limitations we shall show only the results; the derivations will be presented in detail elsewhere. ${ }^{7,8}$ So, the reaction rate and the antineutrino emissivity rate per baryon for the decay process $d \rightarrow u+e^{-}+\bar{\nu}_{e}$ are given by

$$
\begin{gathered}
\Gamma_{1}=\frac{3 G_{F}^{2} \cos ^{2} \theta_{C}}{4 \pi^{5}} \int_{0}^{\infty} \frac{\left(\xi_{d}+E_{\bar{\nu}_{e}}\right)^{2}+\pi^{2} T^{2}}{e^{\left(\xi_{d}+E_{\bar{\nu}_{e}}\right) / T}+1} \frac{I\left(\mu_{u}, \mu_{e}, \mu_{d}, E_{\bar{\nu}_{e}}\right)}{e^{-E_{\bar{\nu}_{e}} / T}+1} d E_{\bar{\nu}_{e}}, \\
\varepsilon_{1}=\frac{3 G_{F}^{2} \cos ^{2} \theta_{C}}{4 \pi^{5}} \int_{0}^{\infty} \frac{\left(\xi_{d}+E_{\bar{\nu}_{e}}\right)^{2}+\pi^{2} T^{2}}{e^{\left(\xi_{d}+E_{\bar{\nu}_{e}}\right) / T}+1} \frac{I\left(\mu_{u}, \mu_{e}, \mu_{d}, E_{\bar{\nu}_{e}}\right)}{e^{-E_{\bar{\nu}_{e}} / T}+1} E_{\bar{\nu}_{e}} d E_{\bar{\nu}_{e}},
\end{gathered}
$$

where $\xi_{d}=\mu_{u}+\mu_{e}-\mu_{d}$ and $I\left(\mu_{u}, \mu_{d}, \mu_{u}, E_{s}\right)$ is the angular integral given in Ref. ${ }^{9}$ The rate $\Gamma_{2}$ and the emissivity $\varepsilon_{2}$ for the decay process $s \rightarrow u+e^{-}+\bar{\nu}_{e}$ can be obtained replacing $\mu_{d}$ by $\mu_{s}$ and $\cos ^{2} \theta_{C}$ by $\sin ^{2} \theta_{C}$ in the expression for $\Gamma_{1}$ and $\varepsilon_{1}$.

For the process $u+e^{-} \rightarrow d+\nu_{e}$ we have

$$
\begin{gathered}
\Gamma_{3}=\frac{3 G_{F}^{2} \cos ^{2} \theta_{C}}{4 \pi^{5}} \int_{0}^{\infty} \frac{\left(\xi_{d}-E_{\nu_{e}}\right)^{2}+\pi^{2} T^{2}}{e^{\left(\xi_{d}-E_{\nu_{e}}\right) / T}+1} \frac{J\left(\mu_{u}, \mu_{e}, \mu_{d}, E_{\nu_{e}}\right)}{e^{-E_{\nu_{e}} / T}+1} d E_{\nu_{e}}, \\
\varepsilon_{3}=\frac{3 G_{F}^{2} \cos ^{2} \theta_{C}}{4 \pi^{5}} \int_{0}^{\infty} \frac{\left(\xi_{d}-E_{\nu_{e}}\right)^{2}+\pi^{2} T^{2}}{e^{\left(\xi_{d}-E_{\nu_{e}}\right) / T}+1} \frac{J\left(\mu_{u}, \mu_{e}, \mu_{d}, E_{\nu_{e}}\right)}{e^{-E_{\nu_{e}} / T}+1} E_{\nu_{e}} d E_{\nu_{e}} .
\end{gathered}
$$

Again, the rate $\Gamma_{4}$ and the emissivity $\varepsilon_{4}$ for $u+e^{-} \rightarrow s+\nu_{e}$ can be obtained replacing $\mu_{d}$ by $\mu_{s}$ and $\cos ^{2} \theta_{C}$ by $\sin ^{2} \theta_{C}$ in the last expressions. The total antineutrino 
and neutrino emissivity in a NS are $\varepsilon_{\bar{\nu}_{e}}=\varepsilon_{1}+\varepsilon_{2}$ and $\varepsilon_{\nu_{e}}=\varepsilon_{3}+\varepsilon_{4}$. Finally, for nonleptonic process $u_{1}+d \rightarrow u_{2}+s$ we use

$$
\Gamma_{5}^{d i r}=\frac{9 G_{F}^{2} \sin ^{2} \theta_{C} \cos ^{2} \theta_{C}}{4 \pi^{5}} \int_{m_{s}}^{\infty} \frac{\left(\mu_{d}-E_{s}\right)^{2}+\pi^{2} T^{2}}{e^{\left(\mu_{d}-E_{s}\right) / T}+1} \frac{J\left(\mu_{u}, \mu_{d}, \mu_{u}, E_{s}\right)}{e^{\left(\mu_{s}-E_{s}\right) / T}+1} d E_{s},
$$

where $J\left(\mu_{u}, \mu_{d}, \mu_{u}, E_{s}\right)$ is the angular integral given in Ref. ${ }^{9}$ The rate for the reverse process $u+s \rightarrow u+d$ is related to the rate of the direct process by

$$
\Gamma_{5}^{r e v}=e^{-\left(\mu_{d}-\mu_{s}\right) / T} \Gamma_{5}^{d i r} .
$$

\section{Results and Conclusions}

Considering the rate processes for a NS, we have

$$
\begin{aligned}
& \frac{d Y_{u}}{d t}=\frac{1}{n_{B}}\left[\Gamma_{1}+\Gamma_{2}-\Gamma_{3}-\Gamma_{4}\right], \\
& \frac{d Y_{d}}{d t}=\frac{1}{n_{B}}\left[-\Gamma_{1}+\Gamma_{3}-\Gamma_{5}^{d i r}+\Gamma_{5}^{r e v}\right],
\end{aligned}
$$

where $Y_{i}$ is the abundance of the $i$-species, it is given by $Y_{i}=n_{i} / n_{B}$ and we can relate the particle abundances through the baryon number conservation and charge neutrality,

$$
\begin{aligned}
& Y_{s}=3-Y_{u}-Y_{d}, \\
& Y_{e}=Y_{u}-1 .
\end{aligned}
$$

The temperature variations can be obtained from the first law of thermodynamics,

$$
\frac{d T}{d t}=-\left(9.86 T \sum_{i} \frac{Y_{i}}{p_{F}(i)}\right)^{-1}\left[\left(\mu_{u}+\mu_{d}-\mu_{s}\right) \frac{d Y_{u}}{d t}+\left(\mu_{d}-\mu_{s}\right) \frac{d Y_{d}}{d t}\right]
$$

Integrating the last equations numerically, we can obtain the time evolution of the temperature and the particle abundances as the system appraches to equilibrium. We see significantly increases the temperature $T$ and the strange quark abundance $Y_{s}$ in a timescale of $\sim 10^{-9}$ s and the abundances of the other particles $Y_{u}, Y_{d}, Y_{e}$ decrease. Due to space limitations we shall show only the results; the derivations will be presented in detail elsewhere ${ }^{7,8}$ because the calculations provide the time evolution of the temperature $T$ and the chemical potentials, we are able to determine the relevance of the different weak interaction processes as matter approximate equilibrium.

The neutrino emissivity per baryon is shown in the left panel of Fig. 1 for the decay processes. The emissivity is high during $\sim 10^{-10} \mathrm{~s}$ and is followed by a steep decline to a value five orders of magnitude smaller in a timescale of $\sim 10^{-8} \mathrm{~s}$ and maximum values of the neutrino emissivity per baryon are $10^{10} \mathrm{MeV} / \mathrm{s}$ and $10^{12}$ $\mathrm{MeV} / \mathrm{s}$. On the other hand, the antineutrino emissivity per baryon is shown in the center panel of Fig. 1 for the processes $d \rightarrow u+e^{-}+\bar{\nu}_{e}$ and $s \rightarrow u+e^{-}+\bar{\nu}_{e}$. The emissivity increases during $\sim 10^{-9} \mathrm{~s}$ and is followed by a steep decline, beside the 

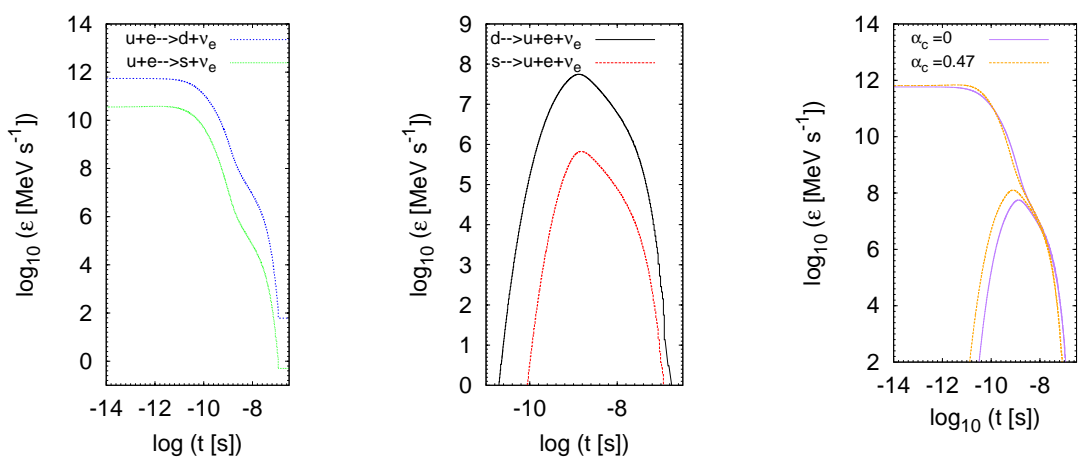

Fig. 1. The reaction rate per unit volume and The neutrino antineutrino emissivity per baryon (left), the antineutrino emissivity per baryon (center) and the total emissivity per baryon (right) as a function of time. We consider $T_{i}=0 \mathrm{MeV}, \mathrm{B}=160 \mathrm{MeV} \mathrm{fm}^{3}$ and $m_{s}=100 \mathrm{MeV}$.

maximum value of the emissivity is between $10^{8} \mathrm{MeV} / \mathrm{s}$ and $10^{6} \mathrm{MeV} / \mathrm{s}$. Finally, the total emissivity per baryon is shown in the right panel of Fig. 1, we see that the total emissivity follows the same behavior each energy separately emissivity. The electron capture reactions are the most important processes that emissivity and the total emissivity increases with $\alpha_{c}$. We have obtained the total energy per baryon, it integrated in time the total emissivity per baryon and if we consider a typical NS with $10^{58}$ baryons, the total emitted energy is around $\sim 10^{53} \mathrm{erg}$. This value is if the same order of the gravitational binding energy of the compact object. Beside, total energy is of the order of the energy of a gamma ray burst (GRB).

\section{References}

1. G. Lugones and O. G. Benvenuto, Phys. Rev. D 58, 83001 (1998).

2. T. do Carmo and G. Lugones, Physica A. 392, 6536 (2013).

3. G. Lugones et al., Astrophys. J. 581, L101 (2002).

4. J. Anand et al., Astrophys. J. 481, 954 (1997).

5. Z. Dai et al., Astrophys. J. 440, 815 (1995).

6. L. Xiang-Jun et al., Commun.Theor. Phys. 49, 1643 (2008).

7. J. A. Rosero and G. Lugones, in preparation.

8. J. A. Rosero and G. Lugones, Nuclear and Particle Physics Proceedings 267-269, 227 (2015).

9. A. Wadhwa et al., J. Phys. G. 21, 1137 (1995). 